\title{
El tratamiento con acarbosa disminuye el riesgo cardiovascular en intolerantes a la glucosa
}

Acarbose treatment and the risk of cardiovascular disease and hipertension in patientes with impaired glucosa tolerance. The STOPNIDDM trial. Chiasson J, Josse R et al. JAMA July 23/30, 2003-Vol 290, 4:486-94.

\section{Objetivo}

Evaluar el efecto del tratamiento con Acarbosa sobre el riesgo cardiovascular e hipertensión arterial en pacientes con intolerancia oral a la glucosa.

Diseño

Ensayo clínico prospectivo, multicéntrico, aleatorizado y controlado con un seguimiento promedio de 3,3 años.

Lugar

Varios hospitales de Canadá, Alemania, Austria, Noruega, Dinamarca, Suecia, Finlandia, Israel y España.

Se incluyeron 1429 pacientes que cumplían criterios de la OMS para intolerancia a la glucosa y que además presentaban glucemia en ayunas de 100 a 140mg/dl.Al ingresar al estudio no debían haber padecido ningún evento cardiovascular en los seis meses previos. Intervención

Acarbosa $300 \mathrm{mg} / \mathrm{día}(\mathrm{n}=714)$ versus placebo $(\mathrm{n}=715)$.A todos los pacientes se les aconsejó bajar de peso o mantenerse en el peso ideal y practicar ejercicio regularmente.Se realizó un seguimiento promedio de 3,3 años y $24 \%$ abandonó el estudio antes de cumplir los tres años (esos pacientes fueron seguidos e incluídos en el análisis por intención de tratar).

Medición de resultados principales

Desarrollo de eventos cardiovasculares mayores (enfermedad coro-

\section{Pacientes}

naria, muerte por enfermedad cardiovascular, insuficiencia cardíaca, accidente cerebrovascular y enfermedad vascular periférica) y de hipertensión arterial.Ver cuadro 1.

Resultados

Cuadro 1.Efecto de la Acarbosa en el desarrollo de eventos cardiovasculares e hipertensión.

\begin{tabular}{|c|c|c|c|c|}
\hline \multirow[t]{2}{*}{ Evento } & \multicolumn{4}{|c|}{$\begin{array}{l}\text { Número de pacientes } \\
\text { con eventos }\end{array}$} \\
\hline & $\begin{array}{l}\text { Acarbosa } \\
(\mathrm{n}=682)\end{array}$ & $\begin{array}{l}\text { Placebo } \\
(n=686)\end{array}$ & $\begin{array}{l}\text { Riesgo relativo ajustado } \\
\text { por otros factores de riesgo } \\
\text { vascular (IC 95\%) }\end{array}$ & NNT \\
\hline Infarto agudo de miocardio & 1 & 12 & \begin{tabular}{|c|}
$0,09(0,01-0,72)$ \\
\end{tabular} & 62 \\
\hline Angina de pecho & 5 & 12 & $0,45(0,16-1,28)$ & \multirow{6}{*}{$\begin{array}{l}\text { No } \\
\text { calculabl }\end{array}$} \\
\hline Revascularización & 11 & 20 & $0,61(0,29-1,26)$ & \\
\hline Muerte cardiovascular & 1 & 2 & $0,55(0,05-6,11)$ & \\
\hline Insuficiencia cardíaca & 0 & 2 & No calculable & \\
\hline Accidente cerebrovascular & 2 & 4 & $0,56(0,10-3,07)$ & \\
\hline Enf. vascular periférica & 1 & 1 & $1,14(0,07-18,30)$ & \\
\hline Cualquier evento vascular & 15 & 32 & $0,46(0,24-0.89)$ & 40 \\
\hline Hipertensión arterial & 78 & 115 & $0,62(0,45-0,86)$ & 19 \\
\hline
\end{tabular}

Conclusiones

NNT:Numero necesario a tratar.

El tratamiento con Acarbosa en los intolerantes a la glucosa se relaciona con una reducción significativa del riesgo de eventos cardiovasculares mayores e hipertensión arterial.

Fuente de financiamiento: Bayer AG.

\section{Comentario}

Este trabajo es el primer ensayo clínico que mostró asociación entre el tratamiento con Acarbosa (que actúa disminuyendo los valores de glucemia postprandial) y la disminución de eventos cardiovasculares mayores e hipertensión arterial, en un subgrupo de pacientes de riesgo cardiovascular mayor que la población general. El estudio UKPDS ${ }^{1}$ había mostrado que el control estricto de la glucemia se asociaba a disminución de complicaciones microvasculares en diabéticos tipo 2 pero no estaba claro si un estricto control glucémico evitaba también los eventos cardiovasculares, la principal causa de muerte en estos pacientes.

Otros trabajos habían postulado a la hiperglucemia, especialmente postprandial, como factor de riesgo cardiovascular independiente en diabéticos y no diabéticos ${ }^{2,3,4}$. Estos estudios postulan que la enfermedad macrovascular comenzaría antes que la diabetes, concepto reforzado por otros ensayos clínicos que demostraron un mayor riesgo de enfermedad cardiovascular en intolerantes a la glucosa en relación a la población genera ${ }^{3,4,5,6,7}$.

El Finnish Diabetes Prevention Study ${ }^{8}$ mostró que se puede prevenir la aparición de diabetes mediante cambios en el estilo de vida en intolerantes a la glucosa y otros dos estudios mostraron que también puede ser prevenida con Metformina ${ }^{9}$ y Acarbosa ${ }^{10}$.El trabajo que demostró esta última asociación fue justamente la primera parte de este trabajo, publicada el año pasado.

Es decir que la diabetes puede prevenirse, pero; ¿pueden prevenirse también las complicaciones cardiovasculares y la muerte en estos individuos "prediabéticos"? Es este el punto que intenta responder la segunda parte del STOP-NIDDM trial, y parece que la respuesta es afirmativa por lo menos en relación a la Acarbosa.Este fármaco disminuye el riesgo de eventos cardiovasculares mayores en intolerantes a la glucosa en un $2,5 \%\left(R_{R A}^{*}\right)$ y sería necesario tratar a 40 intolerantes a la glucosa con Acarbosa por un poco más de tres años para evitar un evento cardiovascular mayor.Debido a los escasos pacientes que fallecieron en este estudio, suponemos que el poder no fue suficiente para detectar diferencias en mortalidad, por lo que esta importante pregunta queda todavía sin respuesta.

Otro dato interesante es que el tratamiento con Acarbosa disminuye en un 5,4\% el riesgo de desarrollar hipertensión arterial (NNT=19). Considerando que los intolerantes a la glucosa tienen alta probabilidad de complicarse y morir de la resultante de sus factores de riesgo vascular, la posibilidad de disminuír el riesgo de hipertensión es un dato muy alentador. Quedan sin embargo varios interrogantes a plantear, todavía no resueltos: ¿Deberíamos efectuar rastreo de los intolerantes a la gluocosa? ¿Con qué método? ¿No estaríamos "enfermando" gente "sana"? ¿Cúal es la línea de corte salud-enfermedad? ¿Es costo-efectivo rastrear y luego tratar con Acarbosa en intolerantes a la glucosa? Recordemos que la intervención sería masiva y que estaríamos tratando a gente cada vez más "sana".¿Cómo le indicamos a un paciente que se considera sano una medicación para prevenir un riesgo "hipotético", sobre todo considerando que el tratamiento con Acarbosa se asoció a una alta tasa de abandono por intolerancia digestiva? Probablemente sean más costo-efectivas las intervenciones sobre el estilo de vida que la administración de Acarbosa, pero debemos recordar que es este fármaco solamente el que ha demostrado disminuír las complicaciones cardiovasculares en los intolerantes a la glucosa y todavía no lo ha hecho la estrategia de cambio en el estilo de vida. Probablemente este trabajo abra camino a nuevos ensayos clínicos que intenten responder estas preguntas.

Conclusiones del comentador: Por ahora, ante nuestro paciente individual intolerante a la glucosa, tenemos, en la Ascarbosa, una opción válida y eficaz en disminuir el riesgo cardiovascular global y el de desarrollar hipertensión, aunque recordando siempre el consejo de cambios de estilo de vida que puede surgir en el futuro como una estrategia más costo-efectiva.

Referencias

1.Stratton et al.Association of glycaemia with macrovascular and microvascular complications of type 2 diabetes (UKPDS 35):prospective observational study.BMJ 2000; $321: 405-412$.

2. Coutinho $\mathrm{M}$ et al. The relationship between glucose and incident cardiovascular events: a metaregression analysis of published data from 20 studies of 95783 individuals followed for 12.4 years. Diabetes Care.1999;22:233-240.

3.Hanefeld et al.Glucose tolerance and cardiovascular mortality:comparison of fasting and 2-hour diagnostic criteria.Arch.Intern.Med.2001;161:397-405.

4. Hanefeld M.et al.Risk factors for myocardial infarction and death in newly detected NIDDM:the diabetes intervention study, 11 years follow-up.Diabetologia.1996;39:1577-1583.

5. Fuller J.et al.Coronary-heart-disease risk and impaired glucose tolerance. Lancet 1980;1:1373-1376

6. Fontbonne a et al.Hipertrygliceridaemia as a risk factor of coronary heart disease mortality in subjectswith impaired glucose tolerance or diabetes. Diabetologia 1989.32.300-304.

7. Tominaga M.et al.Impaird glucose tolerance is a risk factor for cardiovascular disease, but not impaired fasting glucose:the Funagata diabetes study.

7. Tominaga M.et al.Impaird glucose tolerance is a risk factor for cardiovascular disease, but not impaired fasting glucose:the Funagata diabetes study.
8. Tuomilehto J.et al.Prevention of type 2 diabetes mellitus by changes in lifestyle among subjects with impaired glucose tolerance. New Eng J Med 2001

8. Tuomilehto J.et al.Prevention of type 2 diabetes mellitus by changes in lifestyle among subjects with impaired glucose tolerance.New Eng J Med 2001;344:1343-1350 .

9. Diabetes Prevention Program Research Group.Reduction in the incidence of type 2 diabetes with Lifestyle intervention or Metformin.New Eng J Med 2002;346:393-406.

10. Chiasson JL.Et al.Acarbose for prevention of type 2 diabetes mellitus:the STOP NIDDM randomised trial.Lancet 2002;359:2072-2077. 